\title{
OC100: Dietary patterns in 4-18 month old infants: Diet and Nutrition Survey of Infants and Young Children 2011 (DNSIYC)
}

\author{
A. Kokkinopoulou, Y. Brogan and J. Armstrong \\ School of Health and Life Sciences, University Glasgow Caledonian, Glasgow, Scotland
}

Adequate nutrition and infant feeding practices during infancy and early years of childhood are essential for ensuring growth, health and development of children ${ }^{(1)}$. Dietary patterns provide insights into the types of foods being consumed together in the diet. The Diet and Nutrition Survey of Infants and Young Children (DNSIYC) survey is a representative sample $(n 2,683)$ of UK infants and young children aged 4-18 months ${ }^{(2)}$. Principal component analysis (PCA) was applied to the mean intake of a 4-day-dietary diary to derive dietary patterns in 4-8, 9-12 and 13-18 months age groups. Foods with factor loadings above $0 \cdot 3$ were used to characterize the patterns.

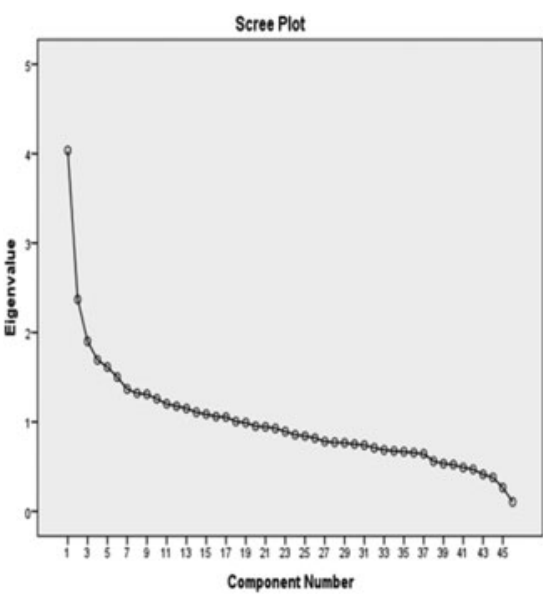

Fig. 1. Scree plot for infants $4-8$ months.

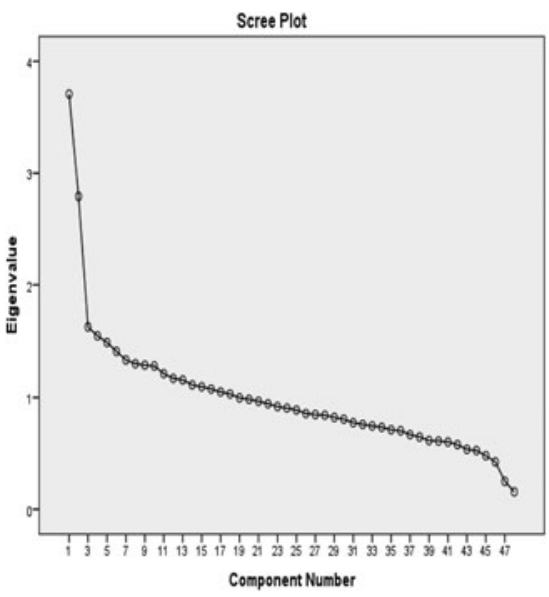

Fig. 2. Scree plot for infants $9-12$ months.

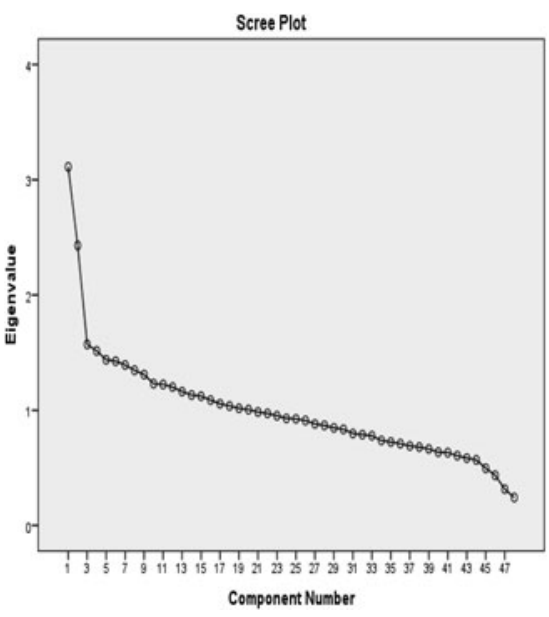

Fig. 3. Scree plot for infants $13-18$ months.

The first dietary pattern in infants aged 4-8 months (Fig. 1) was the High energy/Processed Foods pattern, explaining $8 \cdot 7 \%$ of the variance and included fat spreads, white bread, sugar confectionery, crisps and others. The second, Healthy pattern explained 5.1\% of variance and included oily fish, cheese, white meat, fruits, vegetables and others. The third dietary pattern which explained $4.1 \%$ of the variance was the Commercial Toddler Foods and Beverages pattern which included many commercial toddler foods. In the 9-12 months age group two distinct dietary patterns were observed (Fig. 2). The first dietary pattern was the Healthy pattern and explained $7.7 \%$ of the variance, followed by the High Energy/Processed Foods pattern that explained 5.8\% of the variance. Lastly, two dietary patterns were observed in young children aged 13-18 months (Fig. 3). The High Energy/Processed Foods pattern explained 6.4\% of the variance and the Healthy pattern that explained $5 \%$ of the variance.

Dietary patterns emerge from infancy. The effects of these patterns on growth, development and on long-term outcomes need to be investigated.

1. WHO. Infant and Young Child Feeding. Geneva: WHO Press; 2009. http://www.who.int/nutrition/publications/infantfeeding/9789241597494/en/ (accessed 25 Mar 2014).

2. Department of Health. Diet and Nutrition Survey of Infants and Young Children. http://transparency.dh.gov.uk/2013/03/13/dnsiyc-2011/ (accessed 25 Mar 2014). 